\title{
Wearable activity trackers in cardiovascular research
}

Consumer-grade, wearable activity trackers, or 'wearables', have been used to promote increased fitness in the population, but the integration of the data collected by these devices into basic, translational or clinical research is lacking. In research that begins to provide this integration, Patrick Tan and colleagues found that resting heart rate data is associated with cardiovascular and metabolic disease markers.

The researchers used the Fitbit Charge HR fitness tracker to monitor the physical activity (step counts) and heart rate of 233 healthy volunteers for a median duration of 4 days. Each volunteer answered detailed lifestyle questionnaires and had clinical measurements taken (including weight and blood pressure), as well as undergoing lipidomic profiling.

"This initial work should be regarded as a proof-of-concept study of the value of multi-modality cohort profiling," explains Tan.

Tan and colleagues found that resting heart rate was a better predictor than step counts for seven of nine clinical markers of cardiovascular and metabolic disease, including diastolic blood pressure, triglyceride levels and BMI. The researchers also found step count data could be used to identify relationships between physical activity levels and sphingolipid abundance. Ceramides were previously reported to be negatively associated with physical activity, and this negative association was also observed in this study. New negative associations between other sphingolipids (such as sphingomyelins and glucoslyceramide) and physical activity were also found. "We are planning on extending this strategy to patient cohorts with disease conditions, such as cardiovascular and metabolic diseases," concludes Tan. "Our ultimate goal is to generate multi-modal data signatures that can be used to aid in early detection of disease."

Ivone Leong

ORIGINAL ARTICLE Lim, W. K. et al. Beyond fitness tracking: the use of consumer-grade wearable data from normal volunteers in cardiovascular and lipidomics research. PLoS Biol. 16, e2004285 (2018) 